\title{
Patient Perspectives and Experience with Dalfampridine Treatment in Multiple Sclerosis-Related Walking Impairment: The Step Together Program
}

\author{
Heidi Crayton • Matthew Sidovar • \\ Stanley Wulf • Amy Guo
}

Published online: 5 December 2014

(C) The Author(s) 2014. This article is published with open access at Springerlink.com

\begin{abstract}
Background Dalfampridine extended-release tablets (dalfampridine-ER; in Europe, prolonged-release fampridine, and elsewhere, fampridine modified or fampridine sustained release), $10 \mathrm{mg}$ twice daily, are available for the treatment of improvement of walking in patients with multiple sclerosis, as demonstrated by an increase in walking speed. Ondrug patient perspectives and experiences are valuable to understand and manage this patient population.

Objective The objective of this study was to examine perspectives and experiences of patients receiving dalfampridine-ER in a real-world setting.

Methods Step Together, an ongoing program that captures real-world patient experience with dalfampridine-ER treatment, consists of a survey administered at baseline (before dalfampridine-ER initiation) and at 30 (first followup) and 60 days (second follow-up) after initiation. The survey includes modified versions of the 12-item Multiple Sclerosis Walking Scale (mMSWS-12) and the Sheehan Disability Scale (mSDS) to assess walking ability and functional impairment, respectively.

Results As of September 2013, 2,248 patients participated in the baseline survey and 522 completed both follow-up

Electronic supplementary material The online version of this article (doi:10.1007/s40271-014-0102-z) contains supplementary material, which is available to authorized users.

\section{H. Crayton $(\square)$}

MS Center of Greater Washington, 8320 Old Courthouse Road,

Suite 400, Vienna, VA 22181, USA

e-mail: msdoc1@hotmail.com

M. Sidovar · A. Guo

Acorda Therapeutics, Inc., Ardsley, NY, USA

S. Wulf

Physicians Interactive, Reading, MA, USA
\end{abstract}

surveys (completers). Among the completers, improvements in walking ability and function relative to baseline were significant at both follow-ups as measured by mMSWS-12 and mSDS scores, respectively. Notably, 69-74\% of completers at both follow-ups had improved mMSWS-12 scores, with scores greater than the range considered to be minimally clinically significant. Patients who completed the program expressed satisfaction with overall dalfampridineER treatment, and $69 \%$ indicated that the survey would help them communicate better with their healthcare providers. Conclusion Results highlight the utility of patient-reported outcomes in the assessment of patient perspective and experience, providing a useful supplement to traditional objective measures used in clinical studies.

\section{Key Points for Decision Makers}

Step Together, a patient feedback program, provides insight into the real-world perspectives and treatment experiences of patients who have received dalfampridine extended-release tablets (dalfampridine-ER) for multiple sclerosis (MS)-related walking impairment.

Perceived improvements in walking after dalfampridine-ER treatment were statistically and clinically significant relative to baseline measurements. Perceived improvements were also reported on the impact of MS symptoms on work, social life, and family life activities.

Patients who completed the program reported satisfaction with overall dalfampridine-ER treatment, and $69 \%$ of those who completed expressed that the survey would allow them to better communicate with their healthcare providers. 


\section{Introduction}

People with multiple sclerosis (MS) consider walking impairment to be the most challenging aspect of their disease; walking impairment has been reported to substantially reduce independence in activities of daily living, including the ability to participate in physical and social activities [1]. In a survey conducted in 3,670 people with MS, perception of slower walking speed was consistently associated with progressively reduced quality of life, especially for those domains related to physical function, even after adjustment for demographics and other MSrelated disabilities and symptomatology [2].

While disease-modifying therapies (DMTs) continue to be the mainstay of MS treatment for reducing relapses and slowing disease progression, an integrative approach to patient management that targets symptoms and disabilities has increasingly been emphasized [3-5]. Treatment strategies for walking impairment have been limited primarily to non-pharmacologic therapies, such as exercise and rehabilitation, which may provide short-term benefits $[6$, 7]. In 2010, dalfampridine extended-release tablets (dalfampridine-ER; in Europe, prolonged-release fampridine, and elsewhere, fampridine modified or fampridine sustained release), $10 \mathrm{mg}$ twice daily, were approved by the US Food and Drug Administration for the treatment of improvement of walking in patients with MS, as measured by an increase in walking speed [8].

Two phase III trials, MS-F203 [9] and MS-F204 [10], demonstrated that dalfampridine-ER improved walking speed in a proportion of patients with MS. In a pooled analysis of these trials, improvement from baseline in walking speed, as measured by the timed 25 -foot walk (T25FW) was $25.0 \%$ for those who responded to dalfampridine-ER treatment compared with $7.0 \%$ for dalfampridine-ER non-responders and $6.5 \%$ for the placebo group [11]. In addition to the T25FW, which is a clinicianassessed measure for assessment of treatment effect in walking, patient-rated improvement in walking was also demonstrated using the 12-item Multiple Sclerosis Walking Scale (MSWS-12) [12]. Dalfampridine-ER timed-walk responders reported a change of -6.61 points on the MSWS- 12 compared with -0.32 points for non-responders and 0.69 points for placebo-treated patients [11]; a 4- to 6-point change in MSWS-12 score has been considered to be minimally clinically meaningful [13].

Few studies have been published on patient-reported outcomes of dalfampridine-ER in the real-world setting. In one study of 39 veterans followed for up to 1 year, substantial improvements in self-perceived walking ability were observed as early as 6-8 weeks after starting treatment and were maintained for up to 1 year of follow-up [14].
In another study, a case series of 67 patients followed over a 6-month period showed that clinical benefit varied among patients, with a greater proportion showing benefit on self-reported measures than on clinician-assessed measures [15]. These results suggest that patient-reported outcomes, such as the MSWS-12, may also be useful for assessing improvement in walking in real-world clinical practice.

Despite the association between impaired walking and worsening of quality of life [3], there is often a lack of communication regarding issues with walking between patients and their healthcare providers (HCPs). In a survey of 1,011 MS patients conducted by the North American Research Committee on MS, $39 \%$ of patients reported that they had rarely or never spoken with their HCPs about MSrelated walking problems, regardless of how frequently they had experienced these issues [1].

The Step Together program was initiated with the objective of capturing real-world patient experience on dalfampridine-ER treatment with perspectives on walking, and to provide feedback to the patients' HCPs to facilitate communication. Patient feedback programs such as this may also provide value to other healthcare stakeholders, since real-world patient experience and perspectives are becoming important medical-care outcomes in the healthcare decision-making process [16].

\section{Methods}

The Step Together program, initiated in January 2012 and currently active, is available to patients with MS for whom dalfampridine-ER has been prescribed for the treatment of walking impairment. The current analysis comprises data collected through to 2 September 2013, unless otherwise noted. As of this date, a total of 3,094 HCPs from throughout the USA participated in this program and were engaged in identifying MS patients from their practices who were prescribed dalfampridine-ER for the first time, regardless of insurance coverage. To be eligible for inclusion in the program, adult patients prescribed dalfampridine-ER were required to receive their medication through a single specialty pharmacy. There were no other inclusion or exclusion criteria. Patient participation was entirely voluntary and those who wished to participate were provided the opportunity by the specialty pharmacy at the time of first filling of the prescription. For enrollment, patients were required to call a toll-free telephone number and upon providing consent were included in the program. A full clinical trial informed consent was not required as this was not a clinical trial. Institutional Review Board approval or exemption was not sought for this patient/ caregiver survey study. The primary purpose of the survey 
study was for market research wherein participation by the patients was voluntary and patients were prescribed dalfampridine-ER according to their physician's clinical judgment and practice guidelines independent of the survey. No changes were made to patient treatment due to participation in this survey.

The program consists of a survey that was designed by physician and research staff at Physician Interactive, the survey administrator. The survey consists of questions on patient experience with dalfampridine-ER for administration at three timepoints: baseline (prior to initiating dalfampridine-ER) and at 30 (first follow-up) and 60 days (second follow-up) after initiating treatment. At each follow-up visit, patients were asked if they were still taking dalfampridine-ER; those who were not on the drug were excused from taking that follow-up survey. After the baseline survey, patients could participate in the follow-up surveys either by telephone, via an interactive voiceresponse system, or through the Internet. Following completion of each survey, patients were given a pedometer, a neck cooler, and a US\$10 iTunes gift card, respectively; no incentive was provided for HCP participation. The survey responses were shared as customized patient-specific reports with each patient and their HCP.

For the purpose of this analysis, all patient responses were de-identified and aggregated for evaluation of patientreported outcomes and satisfaction with dalfampridine-ER. The survey (Electronic Supplementary Material Online Resource 1) included demographics, baseline clinical characteristics, utilization of and satisfaction with dalfampridine-ER, and patient-reported outcomes on walking and overall function. The two patient-reported measures used were the MSWS-12 [12] and the Sheehan Disability Scale (SDS) [17], both of which were adapted for use in the current survey.

The MSWS-12 is a disease-specific questionnaire consisting of 12 items that assess the impact of MS on walking over a recall period of 2 weeks using a Likert scale ( $1=$ not at all, $5=$ extremely) [12], with an estimated 4to 6-point change identified as the minimal clinically important difference (MCID) based on data from both the phase III trials and community-based studies [13]. A negative change in MSWS-12 score indicates an improvement. Although all 12 items from the MSWS-12 version 1 were used, the scale was modified (mMSWS-12) for the Step Together program. These modifications consisted of removal of the 2-week recall period, and administration of the scale via either a web- or telephone-based system. Scores on the individual items were summed and transformed to a 0-100 scale, with higher scores indicating greater impact of disease on walking. The scores were stratified by quintiles, since no cut-off values currently exist for defining levels of severity. The total mean score was considered an indicator of the overall impact of MS on walking. The proportions of patients who had achieved improvements from baseline that met the MCID thresholds of 4 and 6 points were also calculated.

The SDS is a measure that assesses functional impairment in three interrelated domains [17]. Using a 0-10 visual analog response $(0=$ not at all, $10=$ extremely), the scale generates four scores: a work disability score, a social life disability score, a family life disability score, and a total score. In the current survey, the SDS was modified to include three questions relating to the impact of MS symptoms on work, social life, and daily activities (i.e., "How much have your MS symptoms disrupted your normal work activities; social activities; daily activities?"). Additional modifications included truncating the responses to a $1-9$ scale $(1=$ not at all disrupted, $9=$ completely disrupted) to allow stratification of levels of disruption as low (1-3), moderate (4-6), and high (7-9).

To assess overall satisfaction with dalfampridine-ER treatment and patient perception of improvement in walking, the following two questions were asked: "Overall, how satisfied are you so far with dalfampridine-ER?" and "How satisfied have you been with how dalfampridine-ER has helped with your walking?" The responses were scored on a 1-9 scale with 1 as "not satisfied" and 9 representing "very satisfied."

To capture information on the reasons for not completing the survey, business reply cards (BRCs) were sent during the last 2 weeks of May 2013 to patients who had missed multiple reminders and didn't complete the followup surveys. This component of the survey reflects data collected through to 5 August 2013. The BRCs included two questions, one on current and intended use of dalfampridine-ER (i.e., "Describe your dalfampridine-ER extended release tablet use") and the other on the reason for not having completed the follow-up (i.e., "Why didn't you take your follow-up survey in the Step Together Program?").

Results from the first and second follow-up periods were compared with those of baseline using the Wilcoxon signed-rank test; $p$ values $<0.05$ were considered statistically significant. Descriptive statistics $[N$, mean, standard deviation (SD)] were calculated for continuous variables, and frequencies and percentages were reported for categorical variables. To examine for associations between improvements in mMSWS-12 score and improvements in normal work and normal social and daily activities, Spearman rank-order correlation coefficients $\left(r_{\mathrm{s}}\right)$ were used. Coefficient values of $0.1,0.3$, and 0.5 were interpreted as small, moderate, and high, respectively. All analyses were performed using the Statistical Package for Social Sciences (SPSS ${ }^{\circledR}$, SPSS V 10.0.2 SPSS Inc, Chicago, IL) [18]. 


\section{Results}

\subsection{Patient Demographics and Clinical Characteristics}

As of September 2013, there were 2,324 dalfampridineER-naïve patients enrolled in the program. Of the enrollees, 2,248 $(96.7 \%)$ patients participated in the baseline survey (baseline group), 402 (17.3\%) completed the first follow-up but not the second follow-up, and $522(22.5 \%)$ completed both follow-up surveys (completer group). On average, patients took $42.0 \pm 18.5$ days to complete the first follow-up and $86.0 \pm 53.4$ days for the second followup after the baseline survey. Demographics and clinical characteristics were similar between the baseline and completer groups (Table 1): $73 \%$ female, mean age 50 years, $42 \%$ had MS $>10$ years; approximately $80 \%$ of patients were using DMTs, of which the most common were glatiramer acetate $(\sim 22 \%)$ and interferon- $\beta-1 \mathrm{a}$ $(\sim 21 \%)$.

Table 1 Demographic and clinical characteristics of enrolled patients who participated in the Baseline survey and completed both follow-ups

\begin{tabular}{|c|c|c|}
\hline & $\begin{array}{l}\text { Baseline } \\
(N=2,248)\end{array}$ & $\begin{array}{l}\text { Completers } \\
(n=522)\end{array}$ \\
\hline $\begin{array}{l}\text { Age, years, mean } \pm \text { SD } \\
\text { (range) }\end{array}$ & $\begin{array}{c}50 \pm 10.8 \\
(19-96)\end{array}$ & $\begin{array}{c}50 \pm 10.0 \\
(19-76)\end{array}$ \\
\hline \multicolumn{3}{|l|}{ Age distribution, years, $\%$} \\
\hline$<35$ & 9 & 9 \\
\hline $35-44$ & 19 & 19 \\
\hline $45-54$ & 34 & 37 \\
\hline $55-64$ & 29 & 29 \\
\hline$\geq 65$ & 9 & 6 \\
\hline Female gender, $\%$ & 73 & 73 \\
\hline \multicolumn{3}{|l|}{ MS duration, years, $\%$} \\
\hline$<1$ & 13 & 16 \\
\hline $1-5$ & 23 & 22 \\
\hline $6-10$ & 20 & 18 \\
\hline$>10$ & 42 & 42 \\
\hline Unsure & 2 & 2 \\
\hline \multicolumn{3}{|c|}{ Disease modifying therapy, $\%$} \\
\hline None & 19 & 20 \\
\hline Interferon beta-1a & 21 & 22 \\
\hline Interferon beta- $1 \mathrm{~b}$ & 6 & 7 \\
\hline Glatiramer acetate & 22 & 23 \\
\hline Fingolimod & 7 & 6 \\
\hline Natalizumab & 15 & 15 \\
\hline Other & 10 & 7 \\
\hline $\begin{array}{c}\text { MSWS-12 score, } \\
\text { mean } \pm \text { SD }\end{array}$ & $66.0 \pm 21.8$ & $67.1 \pm 21.1$ \\
\hline
\end{tabular}

MS multiple sclerosis, MSWS-12 12-item Multiple Sclerosis Walking Scale, $N$ sample size, $S D$ standard deviation

\subsection{Patient Perspectives of Treatment Outcome}

\subsubsection{Improvement in Walking}

Among the 522 completers, the mean \pm SD total baseline score for the mMSWS-12 was $67.1 \pm 21.1$. After initiation of treatment, perceived improvements in walking ability were reported by patients, as indicated by mean total scores of $53.1 \pm 23.7$ and $50.4 \pm 24.7$ at the first and second follow-ups, respectively (Fig. 1a). These scores were significantly lower than those at baseline $(p<0.001)$ at both timepoints, and represent changes of -14.0 and -16.7 at the first and second follow-ups, respectively, indicating improvement. Evaluation of patients who achieved the MCID showed that $74 \%$ of patients reported improvement of $\geq 4$-points during each of the two follow-up periods, while $68 \%$ reported improvement of $\geq 6$-points at the first follow-up and $70 \%$ at the second follow-up period (Fig. 1b). Comparable results with those of patients who completed both follow-ups were reported by those patients who completed the first follow-up but not the second $(n=402)$ : mean total mMSWS-12 scores at baseline and at first follow-up were 66.5 and 54.5, respectively; 71 and $65 \%$ reported improvements of $\geq 4$-point and 6-point changes from baseline, respectively.

To determine whether the patient-reported improvement varied with severity of walking impairment at baseline, completers were stratified by quintiles of baseline mMSWS-12 scores (Fig. 1c). The majority of patients ( $>70 \%$ ) reported $\geq 4$-point and $\geq 6$-point changes from baseline in all quintiles except for the first (lowest severity of illness), where $64 \%$ reported $a>4$-point change and $51 \%$ reported a $\geq 6$-point change (Fig. 1c).

\subsubsection{Improvement in Functional Impairment}

At baseline, mean \pm SD scores on modified SDS (mSDS) domains were $6.4 \pm 2.4$ for normal work activities, $6.1 \pm 2.2$ for social activities, and $6.1 \pm 2.0$ for daily activities (Fig. 2a). At the first follow-up period, patients reported significantly less disruption relative to baseline on all mSDS domains $(p<0.001)$ : a reduction of 23,20 , and $20 \%$ for impact on normal work activities, social activities, and daily activities, respectively (Fig. 2a). Small additional improvements were observed at the second follow-up, with mean scores also significantly lower $(p<0.001)$ than baseline by 26,23 , and $25 \%$ for the respective domains (Fig. 2a). Comparable improvement with that of those who completed both follow-ups was reported by those patients who completed the first followup only.

At baseline, approximately $50 \%$ of completers reported scores of 7-9 on all mSDS domains, consistent with a high 

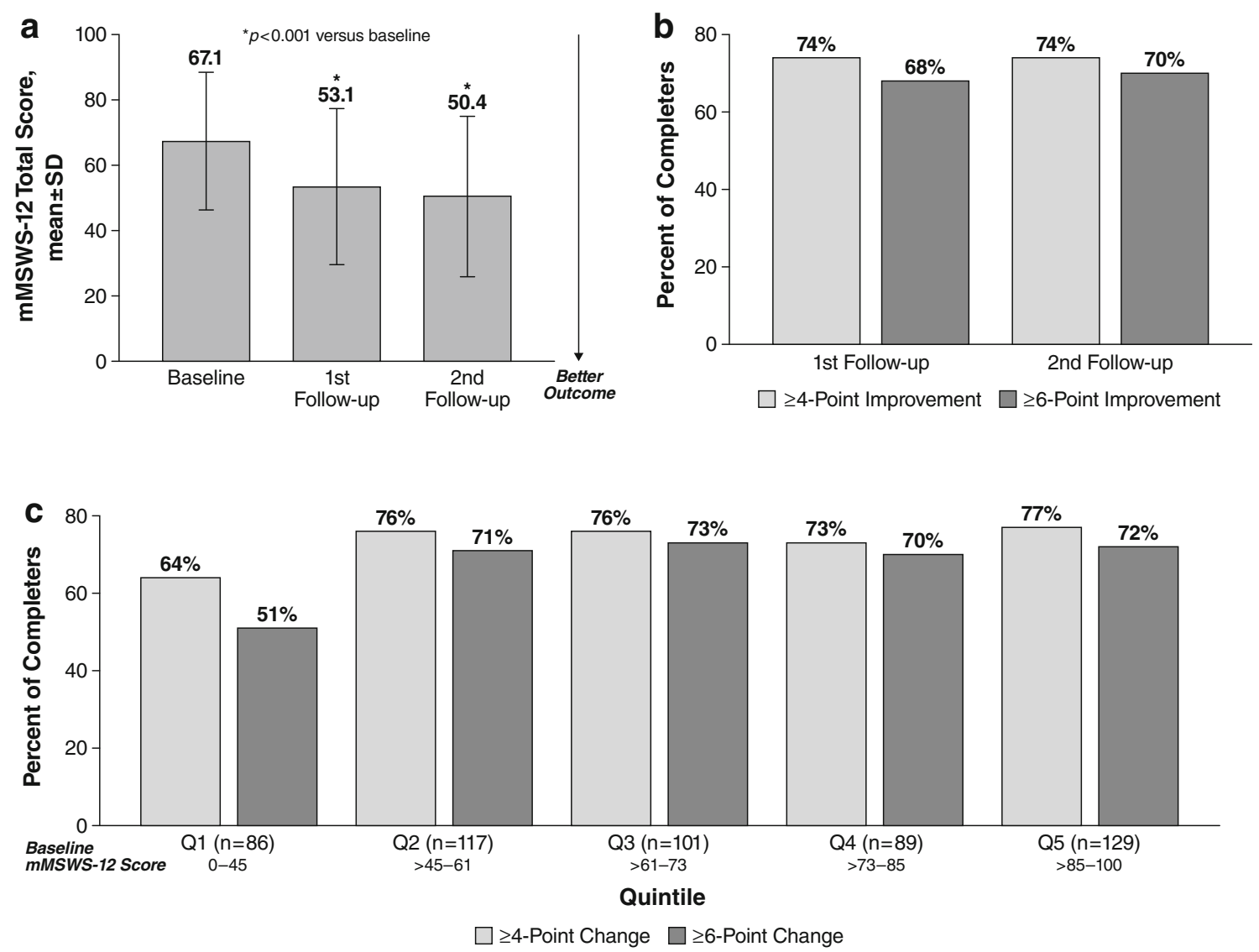

Fig. 1 Patient-reported walking ability as measured by mMSWS in the completers $(n=522)$. a Mean \pm SD score on the mMSWS- 12 . b Proportion of completers achieving $\geq 4$ - and $\geq 6$-point changes from baseline. c Proportion of completers achieving $\geq 4$ - and $\geq 6$-point

level of disruption of daily activities (Fig. 2b). Following treatment with dalfampridine-ER, the proportion of completers who reported high levels of disruption was progressively lower at each follow-up (Fig. 2b), with baseline percentages reduced by approximately half at the second follow-up period (Fig. 2b).

\subsubsection{Improvement in Walking Versus Improvement in Functional Impairment}

Among the completers, change from baseline in total mMSWS-12 scores at both follow-ups were positively and significantly associated with corresponding changes in mSDS domains, indicating that improvement in walking was associated with improvement in functional impairment. At the first follow-up, statistically significant and moderate associations were observed between improvement in walking and improvement in normal work activities $\left(p<0.001, r_{\mathrm{s}}=0.35\right)$, normal social activities $\left(p<0.001, \quad r_{\mathrm{s}}=0.44\right), \quad$ and normal daily activities $\left(p<0.001, \quad r_{\mathrm{s}}=0.46\right)$. At the second follow-up, changes from baseline stratified by baseline mMSWS-12 score quintiles. mMSWS-12 modified 12-item Multiple Sclerosis Walking Scale, $S D$ standard deviation

associations were moderate to strong between improvement in walking and functional domains: normal work $\left(p<0.001, r_{\mathrm{s}}=0.48\right)$, and social $\left(p<0.001, r_{\mathrm{s}}=0.51\right)$ and daily activities $\left(p<0.001, r_{\mathrm{s}}=0.55\right)$.

\subsection{Patient Satisfaction and Communication with Healthcare Providers}

Most patients $(69 \%)$ who completed all three surveys expressed that participating in the survey would help them communicate better with their HCP, $4 \%$ thought it would not help communication, and $27 \%$ were unsure.

Completers expressed moderate satisfaction with dalfampridine-ER on walking and moderate satisfaction overall with treatment: mean $\pm \mathrm{SD}$ satisfaction scores were $5.7 \pm 2.5$ and $5.9 \pm 2.4$, respectively, on a scale of $1-9$. There were no statistically significant differences among quintiles of baseline walking impairment (mMSWS-12 scores) for satisfaction with walking or with overall treatment (Fig. 3). However, overall satisfaction was greater among those with lesser impairment at baseline 
(quintiles 1-3) relative to those with greater impairment (quintiles 4 and 5). Additionally, for each quintile of baseline illness severity, overall treatment satisfaction was
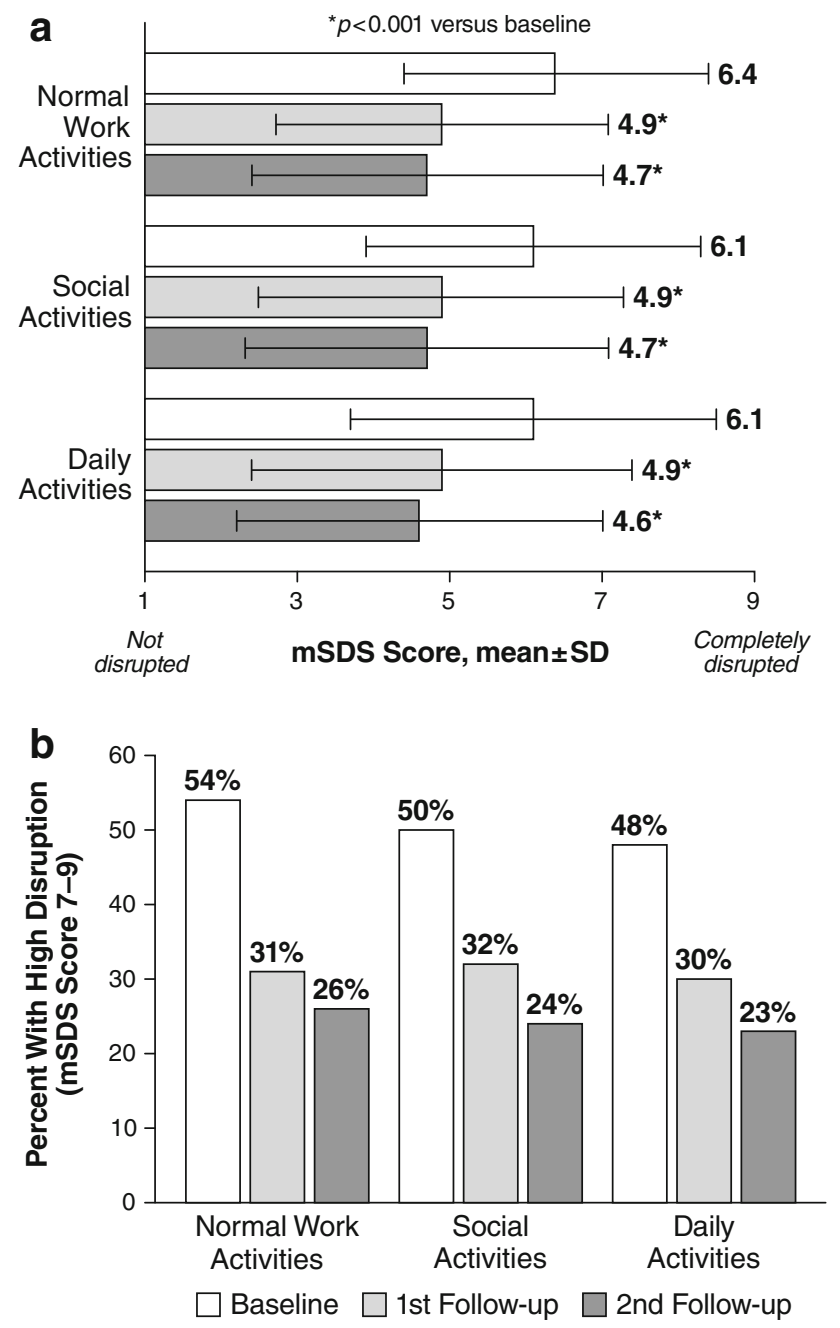

Fig. 2 Functional impairment to normal work, social life, and daily activities as measured by the mSDS in the completers $(n=522)$. a Mean \pm SD scores for baseline and follow-up periods. b Proportion of patients reporting a high level of disruption (score of 7-9 on the mSDS). $m S D S$ modified Sheehan Disability Scale, $S D$ standard deviation higher than satisfaction with walking, but differences were small.

\subsection{Survey Non-Completers}

A total of 635 BRCs were sent to individuals who had participated only in the baseline survey and did not complete the follow-up surveys despite multiple reminders. Of these, 214 were returned; the demographics and clinical characteristics of these patients were comparable with the baseline group. Among those who returned the BRCs, nearly half were patients who viewed dalfampridine-ER treatment favorably, reporting either that they were continuing to take the drug $(47 \%)$ or planned to initiate it (2\%); the remainder consisted of individuals who had discontinued (44\%) or did not plan to initiate treatment (7\%).

\section{Discussion}

Step Together is the first program that provides insight into the real-world perspectives and experiences of patients who initiate dalfampridine-ER treatment for MS-related walking impairment. Based on the results of the enrollees who completed the follow-up surveys, patients perceived that the impact of dalfampridine-ER treatment may extend beyond improvement in walking and may include functional improvements as manifested by less disruption of their normal work, social, and daily activities. Notably, improvement did not vary substantially with baseline severity of walking impairment, as indicated by mMSWS12 scores. These results are consistent with reports from the literature that showed that walking improvement among patients treated with dalfampridine-ER was independent of demographic variables, disease characteristics, and level of disability [11, 19].

The response rates in the current program were similar to those observed in other patient feedback surveys administered in many chronic disease states. For example,
Fig. 3 Patient-reported satisfaction with dalfampridine extended-release treatment on walking and overall satisfaction scores (mean $\pm \mathrm{SD}$ ) by baseline mMSWS-12 score among completers $(n=522)$.

Dalfampridine-ER dalfampridine extended release tablets, $m M S W S-12$ modified 12-item Multiple Sclerosis Walking Scale, $S D$ standard deviation

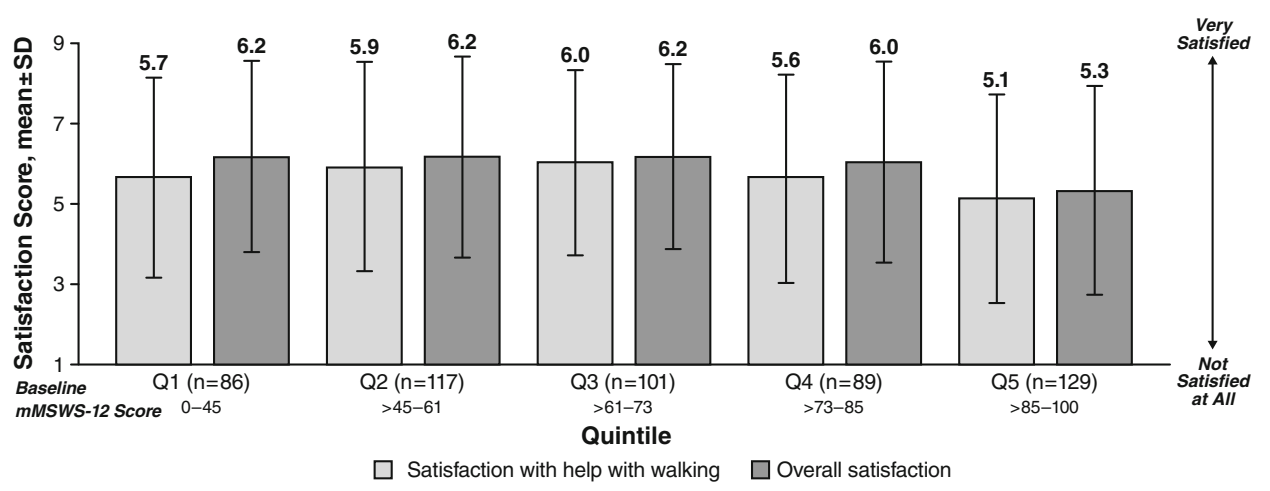


in a parental survey that assessed the treatment effect of lisdexamfetamine dimesylate for pediatric attention deficit hyperactivity disorder, $29.5 \%$ of the baseline participants completed the 6-week follow-up survey [20], whereas, $23.2 \%$ of the baseline survey participants completed both follow-ups in the current program.

While the average magnitude of change in mMSWS-12 in the completer group exceeded 14 points in the follow-up surveys, $68-74 \%$ of patients achieved $\geq 4$ - and 6-point changes that were considered as the MCID for this measure [13]. These changes were observed at both follow-up periods, as well as in all mMSWS-12 baseline quintiles. Furthermore, improvements observed at the first follow-up were maintained at the second follow-up. Both the magnitude of change on the mMSWS-12 and the proportion of patients achieving the MCID were greater in the current program than the changes reported in the dalfampridine-ER clinical studies. In the MS-F203 and MS-F204 studies, 35 and $42.9 \%$ of those treated with dalfampridine-ER were considered as timed-walk responders (placebo: 8 and $9.3 \%$, respectively), based on consistency of walking speed improvement, as measured by T25FW. Timed-walk responders, regardless of treatment assignment, showed greater changes in MSWS-12 scores from baseline relative to non-responders in both studies: -6.84 and -6.04 versus -0.05 and -0.85 in MS-F203 and MS-F204, respectively [9-11]. The results from these clinical trials were consistent with improvements of approximately 11 points on the MSWS-12 that have been reported in clinical practice [14, 15]. In particular, using a case series, Prugger and Berger [15] reported higher proportions of patients as treatment responders based on the response definition of MSWS-12 relative to the T25FW. Although in that study an MSWS12 responder was based on any improvement in MSWS-12 score, the magnitude of the improvement as reported by an 11-point change after 4 weeks of treatment among those who remained on treatment was similar to that observed in the current program. These observations suggest that greater benefits can be obtained from patient-reported outcome scales in the clinical practice relative to what may be expected using clinician-assessed measures in clinical trials. While it is also likely that bias is present in realworld data, the reasons for this observed difference warrant further investigation. The utility of patient-reported outcomes for use in patients treated with dalfampridine is also supported by two recent studies showing benefits in domains of patient-reported quality of life [21, 22].

Overall satisfaction with treatment was consistently higher than satisfaction with walking in all quintiles of baseline impairment, although the differences were small. This relatively greater overall satisfaction with treatment than with walking suggests that patients treated with dalfampridine-ER might be perceiving benefits of treatment that are not captured by walking assessments alone, a possibility previously mentioned in the literature [23]. While quality of life has been assessed in people with MS, the impact of dalfampridine-ER treatment on the functional domains of work and social activities has not been previously reported. Thus, it is of interest to note that improvements in mMSWS-12 scores were paralleled by improvements in mSDS functional domains as indicated by less disruption to normal work, social, and daily activities. The impact of dalfampridine-ER treatment was further strengthened by moderate to strong associations that were observed at both follow-ups, suggesting that improvement in walking may reflect in performance activities.

\subsection{Limitations}

This study reflects real-world utilization of dalfampridineER and reports patient perspectives pertaining to treatment outcomes. Such data are valuable for informing patients, HCPs, and healthcare stakeholders regarding the clinical expectations and outcomes that may be associated with treatment beyond a controlled clinical trial setting.

However, there are several limitations to this analysis. Participants enrolled in the program voluntarily; therefore, selection bias may have been a factor, as patients who completed all three surveys might be different from those who did not. As with all such surveys from patient feedback programs [24-26], the potential for selection bias could arise as patients who had chosen to participate might have had characteristics and outcomes different from those who had not; no information is available on patients who chose not to participate and therefore it was not possible to determine the extent of selection bias that may have impacted this analysis. Although the demographics and baseline severity of walking impairment captured through BRCs were similar in completers and non-completers, other relevant differences might exist between these subgroups that have not been captured. To reduce this bias, further studies should take into account any potential differences in MS patients on dalfampridineER who choose to enroll in the program versus those who do not.

Because the survey was initiated to capture patient perceptions on dalfampridine-ER treatment on walking in order to facilitate communication with their HCPs, it focused primarily on walking outcomes and this may have limited the assessment of other treatment benefits. In addition, the assessment was based on changes from baseline; hence, there were no comparator groups in the program design.

Another limitation is that, although the goal was to follow up with the participants at 30 and 60 days after treatment initiation, the actual duration on dalfampridine- 
ER among the survey respondents varied by \pm 2 weeks for the first follow-up and by as much as 4 weeks for the second follow-up. Thus, it cannot be ruled out that differences between those who responded early versus those who responded late to either of the follow-ups may have affected the results.

Patients had an equal opportunity to report negative and positive responses to the treatment; nonetheless, the responses were subjective and the extent to which patients may have given socially desirable responses is not known. Additionally, this program not only modified the instruments from their existing forms, but also allowed for completion of the survey by either telephone or Internet. Neither the original MSWS-12 and SDS nor their modified versions used in the current study have been validated for administration via telephone or Internet, and these modifications and methods of administration may also have affected the results.

\section{Conclusions}

In this real-world survey, patients reported a clinically and statistically significant association between dalfampridineER treatment and walking improvement, as well as improvements on normal work, social, and daily activities compared with baseline. Patients who completed all three surveys expressed moderate satisfaction with overall dalfampridine-ER treatment, and the majority indicated that participating in the survey would help them communicate better with their HCPs. Notwithstanding the limitations of this survey, the results highlight the utility of patientreported outcome scales in the assessment of patient perspective and experience, providing a useful supplement to traditional objective measures used in clinical studies and practice.

Acknowledgments $\mathrm{HC}$ contributed to data review, interpretation of results, and manuscript development; SW contributed to survey design, data analysis, and manuscript development; MS and AG contributed to data review, interpretation of results, and manuscript development. Funding support for this research was provided by Acorda Therapeutics, Inc. Heidi Crayton has received consulting fees and honorarium from and has been on speakers bureaus for Acorda Therapeutics, Inc. Matthew Sidovar and Amy Guo are employees of Acorda Therapeutics, Inc., and have received stock and/or stock options as part of compensation. Stanley Wulf is a consultant to Acorda Therapeutics, Inc., and was an employee of Infomedics at the time of manuscript development, which has been acquired by Physicians Interactive. Infomedics was funded by Acorda Therapeutics, Inc. to run the study.

The authors thank Bagi Ravishankar, PhD, of Acorda Therapeutics, Inc., and Cathy Counsell of Physicians Interactive for their assistance with manuscript development. Editorial assistance was provided by The Curry Rockefeller Group, LLC, Tarrytown, NY, USA, which was supported by Acorda Therapeutics, Inc., Ardsley, NY, USA.
Open Access This article is distributed under the terms of the Creative Commons Attribution Noncommercial License which permits any noncommercial use, distribution, and reproduction in any medium, provided the original author(s) and the source are credited.

\section{References}

1. LaRocca N. Impact of walking impairment in multiple sclerosis: perspectives of patients and care partners. Patient. 2011;4:189-201.

2. Kohn CG, Baker WL, Sidovar MF, et al. Walking speed and health-related quality of life in multiple sclerosis. Patient. 2014;7:55-61.

3. Cohen BA. Identification, causation, alleviation, and prevention of complications (ICAP): an approach to symptom and disability management in multiple sclerosis. Neurology. 2008;71(24 Suppl 3):S14-20.

4. Samkoff LM, Goodman AD. Symptomatic management in multiple sclerosis. Neurol Clin. 2011;29:449-63.

5. de Sa JC, Airas L, Bartholome E, et al. Symptomatic therapy in multiple sclerosis: a review for a multimodal approach in clinical practice. Ther Adv Neurol Disord. 2011;4:139-68.

6. Brown TR, Kraft GH. Exercise and rehabilitation for individuals with multiple sclerosis. Phys Med Rehabil Clin N Am. 2005;16: 5113-555.

7. Khan F, Turner-Stokes L, Ng L, et al. Multidisciplinary rehabilitation for adults with multiple sclerosis. Cochrane Database Syst Rev. 2007;(2):CD006036.

8. Ampyra $^{\circledR}$ (dalfampridine) extended release (ER) tablets [prescribing information]. Ardsley: Acorda Therapeutics, Inc.; 2014.

9. Goodman AD, Brown TR, Krupp L, et al. Sustained release of oral fampridine in multiple sclerosis: a randomised, double-blind, controlled trial. Lancet. 2009;373:732-8.

10. Goodman AD, Brown TR, Edwards KR, et al. A phase 3 trial of extended release oral dalfampridine in multiple sclerosis. Ann Neurol. 2010;68:494-502.

11. Goodman AD, Brown TR, Schapiro RT, et al. A pooled analysis of two phase 3 clinical trials of dalfampridine in patients with multiple sclerosis. Int J MS Care. 2014;16:153-60.

12. Hobart JC, Riazi A, Lamping DL, et al. Measuring the impact of MS on walking ability: the 12-item MS Walking Scale (MSWS12). Neurology. 2003;60:31-6.

13. Hobart J. Prolonged-release fampridine for multiple sclerosis: was the effect on walking ability clinically significant? [abstract]. Mult Scler. 2010;16(Suppl):S72.

14. Cameron MH, Fitzpatrick M, Overs S, et al. Dalfampridine improves walking speed, walking endurance, and community participation in veterans with multiple sclerosis: a longitudinal cohort study. Mult Scler. 2013;20:733-8.

15. Prugger M, Berger T. Assessing the long-term clinical benefit of prolonged-release fampridine tablets in a real-world setting: a review of 67 cases. Patient Relat Outcome Meas. 2013;4:75-85.

16. Ahmed F, Burt J, Roland M. Measuring patient experience: concepts and methods. Patient. 2014;7:235-41.

17. Sheehan DV, Harnett-Sheehan K, Raj BA. The measurement of disability. Int Clin Psychopharmacol. 1996;11(Suppl 3):89-95.

18. Cohen J. Statistical power analysis for the behavioral sciences. 2nd ed. Hillsdale: Lawrence Erlbaum Associates; 1988.

19. Henney HR III, Blight A. Walking impairment in patients with multiple sclerosis- a new therapeutic approach and clinical potential of dalfampridine extended release tablets. Degener Neurol Neuromusc Dis. 2012;2:53-64. 
20. Antonucci D, Kunins C, Manos M, et al. Assessing effects of treatment with lisdexamfetamine dimesylate for pediatric ADHD using a parental survey. CNS Spectr. 2010;15:248-56.

21. Lycke J, Short C, Gasperini C, et al. Effect of prolonged-release fampridine treatment on walking ability and balance in patients with multiple sclerosis: results of the randomized double-blind MOBILE study [poster no. 101]. 66th Annual Meeting of the American Academy of Neurology; 26 Apr-3 May 2014; Philadelphia.

22. Sorensen PS, Macdonell R, Pozzilli C, et al. The effect of prolonged-release fampridine treatment on health-related quality of life outcomes after 1 year: results from the ENABLE study [poster]. Joint Congress of European Neurology; 31 May-3 Jun 2014; Istanbul.
23. Schapiro RT, Bethoux F, Brown TR, et al. Open-label extension patient retention rates with dalfampridine extended release tablets in multiple sclerosis. [abstract]. Mult Scler. 2012;18(4 Suppl): 477.

24. Stonecipher K, Perry HD, Gross RH, Kerney DL. The impact of topical cyclosporine A emulsion $0.05 \%$ on the outcomes of patients with keratoconjunctivitis sicca. Curr Med Res Opin. 2005;21:1057-63.

25. Kerney DL, Paradis D, Brunton S. Patient perceptions of insulin detemir as reported through patient telephone surveys. Curr Med Res Opin. 2007;23:2043-9.

26. Kerney DL, Ford RO, Gotz V. Self-reported participant experience with desonide hydrogel in the treatment of mild to moderate atopic dermatitis. Cutis. 2011;88:18-24. 